\title{
Adsorption of Guaiacol on Fe (110) and Pd (111) from First
}

\section{Principles}

Alyssa J. R. Hensley ${ }^{a}$, Yong Wang ${ }^{a, b}$, and Jean-Sabin McEwen ${ }^{a, c, d *}$

${ }^{\mathrm{a}}$ The Gene \& Linda Voiland School of Chemical Engineering and Bioengineering, Washington State University, Pullman WA 99164

${ }^{\mathrm{b}}$ Institute for Integrated Catalysis, Pacific Northwest National Laboratory, Richland, WA, 99352

${ }^{c}$ Department of Physics and Astronomy, Washington State University, Pullman, WA 99164

${ }^{\mathrm{d}}$ Department of Chemistry, Washington State University, Pullman, WA 99164

*Corresponding author: Jean-Sabin McEwen; 509-335-8580 (phone), 509-335-4806 (fax), js.mcewen@wsu.edu

\section{Abstract}

The catalytic properties of surfaces are highly dependent upon the effect said surfaces have on the geometric and electronic structure of adsorbed reactants, products, and intermediates. It is therefore crucial to have a surface level understanding of the adsorption of the key species in a reaction in order to design active and selective catalysts. Here, we study the adsorption of guaiacol on Fe (110) and Pd (111) using dispersion corrected density functional theory calculations as both of these metals are of interest as hydrodeoxygenation catalysts for the conversion of bio-oils to useable biofuels. Both vertical (via the oxygen functional groups) and horizontal (via the aromatic ring) adsorption configurations were examined and the resulting adsorption and molecular distortion energies showed that the vertical sites were only physisorbed while the horizontal sites were chemisorbed on both metal surfaces. A comparison of guaiacol's horizontal adsorption on $\mathrm{Fe}$ (110) and Pd (111) showed that guaiacol had a stronger adsorption 
on $\mathrm{Pd}$ (111) while the Fe (110) surface distorted the C-O bonds to a greater degree. Electronic analyses on the horizontal systems showed that the greater adsorption strength for guaiacol on Pd (111) was likely due to the greater charge transfer between the aromatic ring and the surface $\mathrm{Pd}$ atoms. Additionally, the greater distortion of the $\mathrm{C}-\mathrm{O}$ bonds in adsorbed guaiacol on $\mathrm{Fe}(110)$ is likely due to the greater degree of interaction between the oxygen and surface Fe atoms. Overall, our results show that the $\mathrm{Fe}(110)$ surface has a greater degree of interaction with the functional groups and the Pd (111) surface has a greater degree of interaction with the aromatic ring.

\section{Keywords}

Density Functional Theory; Guaiacol Adsorption; Fe (110); Pd (111); van der Waals Corrections

\section{Introduction}

Bimetallic surfaces have many applications as catalysts, from petroleum refining[1] to catalytic converters[2] to Fischer-Tropsch catalysts[3] to methanol fuel cells[4], due to their tunable properties which produce surfaces with superior performance as compared to monometallic surfaces. However, to better optimize and design these bimetallic surfaces, a detailed knowledge of the inter- and intra- surface interactions is necessary for both the bimetallic surface and its monometallic constituents. The application of surface science techniques to gain insight into the bimetallic and monometallic structure and electronic states during various chemical processes has been shown to be of great importance to the design and optimization of catalysts by the Somorjai research group.[5-9]

Of particular interest to our group is elucidating the inter- and intra- surface interactions for Fe based bimetallic catalysts. Recent work on Fe based bimetallic catalysts has shown that these catalysts have a high activity for the hydrodeoxygenation (HDO) of bio-oil oxygenates (e.g. phenolics and furanics),[10-13] a key step in transforming pyrolysis bio-oils into more 
useable biofuel. While Fe alone has been shown to be highly selective for the HDO of phenols, $[10,11]$ it is known to easily form oxides and carbides, quickly deactivating under reaction conditions.[14] However, this surface deactivation can be avoided by doping Fe catalysts with small amounts of precious metals (e.g. Pd or Pt), and such catalysts have shown a high activity for the HDO of phenols, such as guaiacol and m-cresol, producing aromatic hydrocarbon products with a high selectivity.[10, 11]

While these Fe based bimetallic catalysts look promising as HDO catalysts, knowledge of the complex inter- and intra- surface interactions which lead to the high catalytic activity is lacking. Our previous work has shown that the adsorption of aromatics is weakened on model $\mathrm{Pd} / \mathrm{Fe}$ surfaces as compared to both model Fe and Pd surfaces due to the change in Pd's electronic structure in the $\mathrm{Pd} / \mathrm{Fe}$ system.[10, 15] Further examination of the adsorption and HDO mechanism of phenol on monometallic Fe (110) and Pd (111) surfaces showed that the high HDO activity of the Fe catalysts and ring saturation activity of Pd catalysts is likely due to the stronger charge transfer between the oxygen functional group and surface Fe and the aromatic ring and surface Pd.[16, 17] These results strongly suggest that Fe is the active site for the HDO of phenolics on $\mathrm{Pd} / \mathrm{Fe}$ surfaces. As for the role of $\mathrm{Pd}$ in these bimetallic catalysts, several studies on the reducibility of $\mathrm{Fe}_{2} \mathrm{O}_{3}$ doped with $\mathrm{Pd}$ and the stability of the $\mathrm{Pd} / \mathrm{Fe}$ surface under steam exposed has shown that the Pd dopant likely maintains the high HDO activity of the Fe surface by protecting said surface from deactivation via oxidation. $[11,18]$ This work connects to that of the Somorjai research group as they showed that doping Fe single crystal surfaces with potassium and aluminum oxide enhances the activity of Fe for ammonia synthesis by decreasing the adsorption energy of ammonia and maintaining an active Fe surface structure under reaction 
conditions, $[19,20]$ together suggesting that the relatively abundant metal Fe can be easily functionalized to produce highly active catalysts.

While our previous work has elucidated many of the inter- and intra- surface interactions in the Fe based bimetallic catalysts that contribute to the high HDO activity of said catalysts, much is still unknown in this area. In this study, we examine the effect of complex functional groups on the adsorption of phenolics on the Fe (110) and Pd (111) surfaces, using guaiacol as the model phenolic compound as it is a stable bio-oil compound under fast pyrolysis conditions.[10] Furthermore, as the performance of the noble metal promoted-Fe based bimetallic catalysts are experimentally evaluated in comparison to monometallic catalysts formed from the same components, $[10,11]$ the adsorptive characterization of the pure metal surfaces is necessary in order to provide a benchmark against which the atomistic interactions occurring at the bimetallic surfaces can be evaluated and better understood. In addition to providing a benchmark, understanding the adsorption behavior of model HDO compounds can lead to a deeper understanding of the inter- and intra- electronic interactions that occur during aromatic adsorption which can be connected, at least qualitatively, to the selectivities of the monometallic catalysts. Overall, this work shows that the Fe surface electronically interacts to a greater degree with adsorbed guaiacol's functional groups while Pd has a stronger interaction with the aromatic carbon, which is consistent with the catalytic performance of these two metals. Additionally, this work furthers the characterization of aromatics adsorption on metal surfaces, providing additional theoretical benchmarks and expanding our knowledge of surface to adsorbate interactions.[21] 


\section{Methods}

The theoretical calculations presented in this work were performed using the Vienna $A b$ Initio Simulation (VASP) package.[22, 23] The core electrons were treated using VASP's projector augmented waves (PAW)[24, 25] in order to solve the Kohn-Sham equations. The initial density functional theory (DFT) calculations (without van der Waals corrections) were performed using the generalized gradient approximation (GGA) which was parameterized with the Perdew-Wang 91 (PW91) functional.[26, 27] After the most favorable configurations for the parallel and perpendicular adsorption of phenol on Fe (110) and Pd (111) were identified using the PW91 functional, these most favorable configurations were examined using the optB88$\operatorname{vdW}[28,29]$ exchange-correlation functional to account for van der Waals corrections which has shown to strongly affect the adsorption energies of both physisorbed and chemisorbed species. $[30,31]$ When using the optB88-vdW, we switched to the Perdew, Burke, and Ernzerhof (PBE) $[32,33]$ functional to generate the PAW core potentials as we found that the adsorption energy results for the optB88-vdW functional with the PW91 generation of the PAW core potentials varied significantly with minor changes to the model's vacuum thickness. All other parameters were identical to those used in our previous work studying the adsorption of phenol on $\mathrm{Fe}(110)$ and $\mathrm{Pd}(111) .[16]$ In the density of states analyses, the energy levels were normalized such that the Fermi level was set to zero. For gas phase guaiacol, the Fermi level was taken to be halfway between the HOMO and LUMO states, similar to the work of Mittendorfer, et al.[34]

All of the studied adsorption configurations of phenol on the surfaces were compared using the adsorption energy as defined by: $E_{a d s}=E_{\text {adsorbates }+ \text { surface }}-E_{\text {surface }}-E_{\text {adsorbate }}$, where $E_{\text {adsorbates }+ \text { surface }}, E_{\text {surface }}$, and $E_{\text {adsorbate }}$ are the total energies of the adsorbed surface system, the 
clean surface, and the gas phase adsorbates for the given system. Each adsorption site was also evaluated using the distortion energy which is the energy the adsorbate molecule gains due to its geometric distortion from the interaction with the metal surface.[35] The distortion energy will be calculated according to: $E_{\text {dist }}=E_{\text {Molecule }}^{\text {Distorted Geometry }}-E_{\text {Molecule }}^{\text {Free Gas Geometry }}$, where $E_{\text {Molecule }}^{\text {Distorted Geometry }}$ and $E_{\text {Molecule }}^{\text {Free Gametry }}$ are the energies of phenol in the gas phase with its optimized geometry and the distorted geometry caused by interaction with the surfaces studied here. This energy difference quantifies the degree to which the adsorbate is distorted in a single value.

The electronic exchange that occurred upon the adsorption of guaiacol on each surface was examined by calculating the differential charge density as defined by:

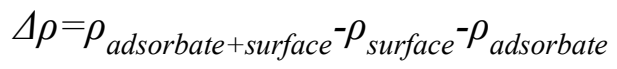

where $\rho_{\text {adsorbate }+ \text { surface }}, \rho_{\text {surface }}$, and $\rho_{\text {adsorbate }}$ are the charge distributions for the optimized structure of the adsorbed system and the surface and gas phase adsorbate fixed in their adsorption geometries. In order to better quantify the electronic interactions observed between guaiacol and the Fe (110) and Pd (111) surfaces, we integrated the absolute value of the differential charge density over the entire unit cell in order to obtain the mean absolute charge transferred between the adsorbate and surface $(Q)$ according to:[36]

$$
Q=1 / 2 \int d \vec{r}|\Delta \rho(\vec{r})|
$$

where $\Delta \rho(\vec{r})$ is the differential charge density of the system. Additionally, the charge transfer between individual atoms within the most favorable adsorption systems was determined using a Bader analysis.[37] Finally, the change in the surface metal's electronic structure upon guaiacol's adsorption was evaluated using the d-band center and width according to:[15, 38]

$$
\varepsilon_{d}=\int_{-\infty}^{\varepsilon_{F e r m i}} E \rho(E) d E / \int_{-\infty}^{\varepsilon_{F e r m i}} \rho(E) d E
$$




$$
w_{d}=\left(\int_{-\infty}^{\varepsilon_{F e r m i}} E^{2} \rho(E) d E / \int_{-\infty}^{\varepsilon_{F e r m i}} \rho(E) d E\right)^{1 / 2}
$$

where $E$ is the given energy, $\rho(E)$ is the density of electronic states at the given energy, and $\varepsilon_{\text {Fermi }}$ is the Fermi level.

\section{Results and Discussion}

\subsection{Gas Phase Guaiacol}

The gas phase configuration of guaiacol was determined by altering the relative positions of the two functional groups, as shown in Figure 1. These results show that the most stable configuration of guaiacol in the gas phase is the structure with the hydroxyl group in a position to hydrogen bond with the oxygen in the methoxy functional group (Figure 1a). This structure was then used for the study of the most favorable adsorption sites for guaiacol on Fe (110) and $\operatorname{Pd}(111)$.

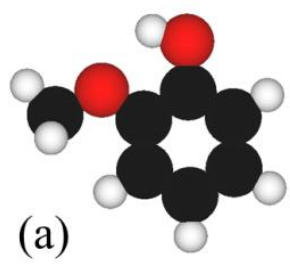

$$
\Delta E=0.00
$$

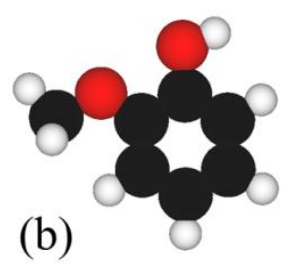

$$
\Delta E=0.18
$$

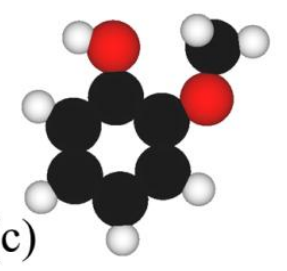

$\Delta E=0.30$

Figure 1. Possible gas phase configurations of guaiacol. The red, black, and white spheres are oxygen, carbon, and hydrogen, respectively.

In order to better understand how guaiacol's orbitals interact with the Fe (110) and Pd (111) surfaces, the electronic configuration for gas phase guaiacol must be investigated. This has been done by calculating the partial density of states (PDOS), as well as the partial charge density distributions for the five highest (most positive) energy orbitals, for the most stable gas phase guaiacol configuration, as shown in Figure 2. For the PDOS, the p-states for the hydroxyl 
oxygen, methyl carbon, and the aromatic carbon para to the methoxy group are shown as both the two oxygen and the six aromatic carbon had similar distributions (PDOS for all the carbon and oxygen atoms are shown in Figure S1). The s-states are not shown as they only have a significant effect on the lowest (most negative) energy orbitals. For the partial charge density distributions, the energy ranges used to calculate said distributions are shown in Table S1.

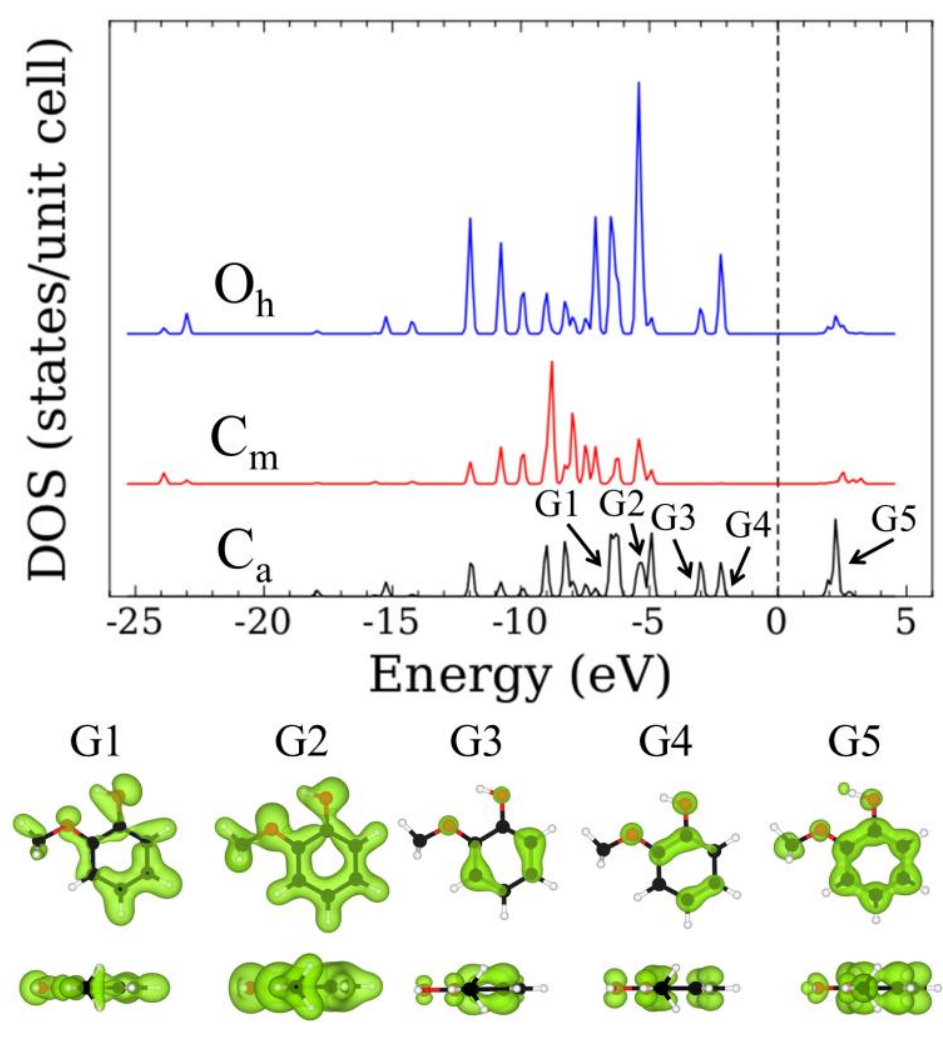

Figure 2. The partial density of states (top) and partial charge density distributions (bottom) for the five highest energy orbitals, labeled with their respective energies, for the most stable gas phase guaiacol configuration. The sphere coloring is identical to Figure 1 and the charge density distribution isosurface was set to 0.01 electrons/Bohr ${ }^{3}$. Also, the Fermi level has been set to zero. An examination of the PDOS for the carbon and oxygen's p-states shows that the HOMO level for guaiacol is dominated by the aromatic carbon and oxygen species. This is mirrored in the partial charge density distribution for the G4 and G3 peaks which show these highest 
occupied orbitals to have a charge distribution in the aromatic carbon's $\pi$ electron cloud and surrounding the oxygen species. Moving lower in energy to the peaks at G2 and G1, it is clear that the charge distribution changes to include both the hydrogen species as well as p-states involved in the bonding between aromatic carbon atoms. The LUMO level at G5 shows contributions from the aromatic carbon, both oxygen species, methyl carbon, and the hydroxyl's hydrogen.

The PDOS and partial charge density distribution results for gas phase guaiacol show similar orbitals to those seen in both gas phase phenol and benzene. The HOMO orbital at -2.2 eV for benzene was shown by Mittendorfer, et al.[34] to have two degenerate charge distributions with both involving the carbon's free p-orbitals. The addition of the oxygen functional groups in both phenol[16] and guaiacol (shown here) splits these degenerate states into two distinct orbitals at $-2.2 \mathrm{eV}$ and $-3.0 \mathrm{eV}, \mathrm{G} 4$ and $\mathrm{G} 5$, respectively. A similar phenomena is seen for the next highest orbital, which for benzene was at $-4.5 \mathrm{eV}[34]$ and for guaiacol can be seen at $-5.1 \mathrm{eV}$ and $-6.4 \mathrm{eV}$ (G2 and G1, respectively). As for the LUMO level, no distribution is given for benzene.[34] However, both the phenol and guaiacol LUMO levels have similar distributions with states available in the aromatic carbon's $\pi$ electron cloud, near the oxygen species, and near the hydroxyl's hydrogen. Based on the similarities between benzene's, phenol's, and guaiacol's electronic structures, it is likely that these species interact in a similar manner to each other on the Fe (110) and Pd (111) surfaces.

\subsection{Vertical Guaiacol Adsorption}

The vertical adsorption of guaiacol on the Fe (110) surface occurs via the oxygen functional group as the $\mathrm{C}-\mathrm{H}$ bonds are known to be repelled by Fe surfaces.[15, 39] This adsorption can occur through the oxygen bonding with the three-fold (3F), long bridge (LB), 
short bridge (SB), or top (T) sites with each site labeled with respect to the hydroxyl group first and the methoxy group second. For guaiacol's vertical adsorption on both Fe (110) and Pd (111), we used the most stable gas phase configuration (Figure 1a) as the adsorption of guaiacol in the second most favorable gas phase guaiacol configuration was found to be less energetically favorable (Figure S2). These configurations are shown in Figure 3a and the resulting adsorption energies (relative to gas phase guaiacol), calculated using the PW91 functional, are shown in Table 1.
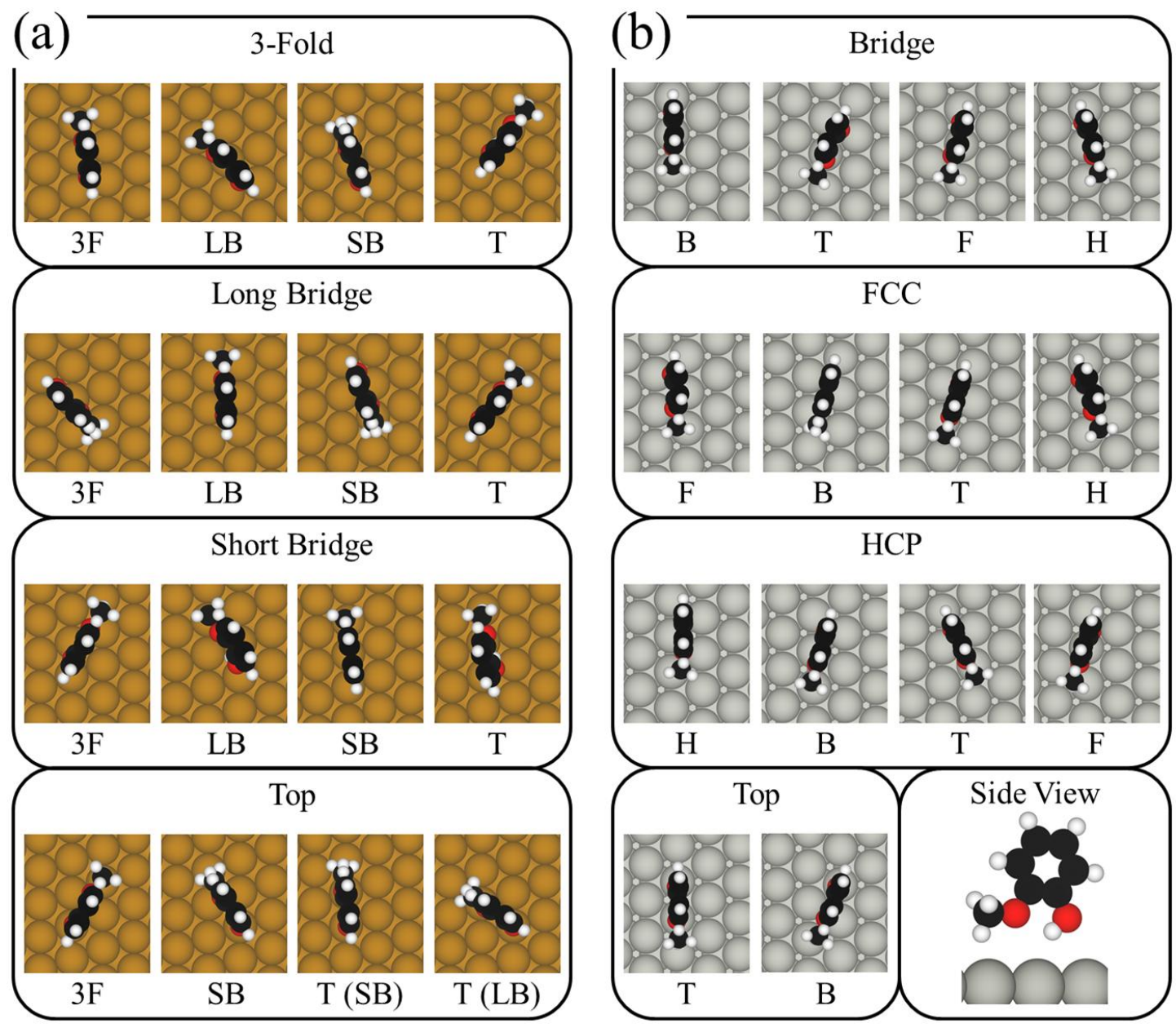

Figure 3. Vertical adsorption sites for guaiacol on the (a) Fe (110) and (b) Pd (111). The gold, silver, red, black, and white spheres are $\mathrm{Fe}, \mathrm{Pd}, \mathrm{O}, \mathrm{C}$, and $\mathrm{H}$, respectively. 
Table 1. Adsorption energies for guaiacol (vertical) on the Fe (110) and $\mathrm{Pd}(111)$ surfaces calculated with the PW91 functional. ${ }^{\text {a }}$ The adsorption energies are shown in the order in which the structures appear in Figure 3. The $U$ symbol marks an adsorption site as unstable.

\begin{tabular}{|c|c|c|c|}
\hline \multicolumn{2}{|c|}{ Fe (110) } & \multicolumn{2}{|r|}{ Pd (111) } \\
\hline Site & $\mathrm{E}_{\mathrm{ads}}(\mathrm{eV})$ & Site & $\mathrm{E}_{\mathrm{ads}}(\mathrm{eV})$ \\
\hline three-fold & $-0.07 /-0.07 / U /-0.10$ & bridge & $-0.11 /-0.13 /-0.11 /-0.11$ \\
\hline long bridge & $U /-0.08 / U /-0.09$ & fec & $-0.12 / U /-0.12 /-0.11$ \\
\hline short bridge & $U /-0.08 /-0.07 /-0.10$ & hep & $-0.12 /-0.12 /-0.11 /-0.10$ \\
\hline top & $-0.08 / U / U / U$ & top & $-0.11 /-0.11$ \\
\hline
\end{tabular}

The most favorable adsorption site for vertical guaiacol on Fe (110) is the 3F-T site with a weak adsorption energy. Also, all stable sites have very similar adsorption strengths and the difference in energy between the strongest and weakest sites is only $0.03 \mathrm{eV}$. Furthermore, the average $\mathrm{C}-\mathrm{C}$ bond length and $\mathrm{C}-\mathrm{H}$ dihedral angle, relative to the plane of the aromatic ring, for the $3 \mathrm{~F}-\mathrm{T}$ site is $1.40 \AA$ and $1^{\circ}$, respectively. In addition, the $\mathrm{C}_{\text {aromatic }} \mathrm{O}$ bond lengths between the oxygen in hydroxyl and methoxy groups and the closest aromatic carbon are $1.37 \AA$ and $1.38 \AA$, respectively.

The vertical adsorption of guaiacol on the Pd (111) surface will also occur through the oxygen functional group as the $\mathrm{C}-\mathrm{H}$ bonds are known to be deflected by the surface.[40, 41] Adsorption through the oxygen group will have the oxygen interacting with the bridge (B), fcc $(\mathrm{F})$, hcp $(\mathrm{H})$, or top $(\mathrm{T})$ sites with each site labeled with respect to the hydroxyl group first and the methoxy group second. These adsorption sites are shown in Figure $3 \mathrm{~b}$ and their corresponding adsorption energies (relative to gas phase guaiacol), calculated with the PW91 functional, are shown in Table 1.

The most favorable adsorption site for vertical guaiacol on $\mathrm{Pd}(111)$ is the B-T site with a weak adsorption energy. All of the stable sites have very similar adsorption strengths and the difference in energy between the strongest and weakest sites is only $0.03 \mathrm{eV}$. The average $\mathrm{C}-\mathrm{C}$ 
bond length and $\mathrm{C}-\mathrm{H}$ dihedral angle, relative to the plane of the aromatic ring, for the $\mathrm{B}-\mathrm{T}$ site is $1.40 \AA$ and $0^{\circ}$, respectively. In addition, the $\mathrm{C}_{\text {aromatic }} \mathrm{O}$ O bond lengths for the hydroxyl and methoxy groups are both $1.37 \AA$.

The most favorable vertical guaiacol adsorption sites on Fe (110) and Pd (111) were studied further using the optB88-vdW functional and the resulting adsorption energies are shown in Table 2. The addition of the van der Waals corrections increased the adsorption energy of vertical guaiacol by $0.4 \mathrm{eV}$ and $0.5 \mathrm{eV}$ on the $\mathrm{Fe}$ (110) and $\mathrm{Pd}$ (111) surfaces, respectively, which is consistent with trends for other aromatic molecules.[15, 16, 31] While there is an increase in the adsorption energy with the addition of van der Waals corrections, the overall strength of adsorption shows vertical guaiacol to be physisorbed on both surfaces. This is further supported by the distortion energy and structural parameters which show almost no deviation from gas phase guaiacol.

Table 2. Adsorption energies, distortion energies, and molecular structure results for guaiacol adsorbed both horizontally and vertically on the Fe (110) and Pd (111) surfaces, as well as the molecular structure results for gaseous guaiacol. ${ }^{a}$ These results were calculated using van der Waals corrections with the optB88-vdW functional.

\begin{tabular}{|c|c|c|c|c|c|c|c|}
\hline \multicolumn{8}{|c|}{ Gas Phase } \\
\hline Site & $\mathrm{E}_{\mathrm{ads}}(\mathrm{eV})$ & $\mathrm{E}_{\text {dist }}(\mathrm{eV})$ & $\mathrm{d}_{\mathrm{C}-\mathrm{C}}(\AA)$ & $\mathrm{d}_{\mathrm{C}-\mathrm{O}}(\AA)$ & $\theta_{\mathrm{C}-\mathrm{H}}\left({ }^{\circ}\right)$ & $\theta_{\mathrm{C}-\mathrm{O}}\left({ }^{\circ}\right)$ & $\mathrm{d}_{\mathrm{A}-\mathrm{M}}(\AA)$ \\
\hline Guaiacol & - & - & 1.40 & $1.37,1.38$ & 0 & 0,6 & - \\
\hline \multicolumn{8}{|c|}{$\mathrm{Fe}(110)$} \\
\hline Site & $\mathrm{E}_{\mathrm{ads}}(\mathrm{eV})$ & $\mathrm{E}_{\mathrm{dist}}(\mathrm{eV})$ & $\mathrm{d}_{\mathrm{C}-\mathrm{C}}(\AA)$ & $\mathrm{d}_{\mathrm{C}-\mathrm{O}}(\AA)$ & $\theta_{\mathrm{C}-\mathrm{H}}\left({ }^{\circ}\right)$ & $\theta_{\text {C-O }}\left({ }^{\circ}\right)$ & $\mathrm{d}_{\mathrm{A}-\mathrm{M}}(\AA)$ \\
\hline $\mathrm{LB} 30^{\circ}-\mathrm{TT}$ & -2.01 & 2.08 & 1.46 & $1.42,1.41$ & 23 & 17,48 & 1.98 \\
\hline $3 \mathrm{~F}-\mathrm{T}$ & -0.47 & -0.02 & 1.40 & $1.37,1.38$ & 1 & 0,6 & 3.36 \\
\hline \multicolumn{8}{|c|}{ Pd (111) } \\
\hline Site & $\mathrm{E}_{\mathrm{ads}}(\mathrm{eV})$ & $\mathrm{E}_{\mathrm{dist}}(\mathrm{eV})$ & $\mathrm{d}_{\mathrm{C}-\mathrm{C}}(\AA)$ & $\mathrm{d}_{\mathrm{C}-\mathrm{O}}(\AA)$ & $\theta_{\mathrm{C}-\mathrm{H}}\left({ }^{\circ}\right)$ & $\theta_{\mathrm{C}-\mathrm{O}}\left({ }^{\circ}\right)$ & $\mathrm{d}_{\mathrm{A}-\mathrm{M}}(\AA)$ \\
\hline $\mathrm{B} 30^{\circ}-\mathrm{BB}$ & -2.27 & 1.18 & 1.45 & $1.37,1.36$ & 22 & 20,23 & 2.18 \\
\hline B-T & -0.65 & 0.00 & 1.40 & $1.37,1.37$ & 1 & 0,6 & 2.66 \\
\hline $\begin{array}{l}\text { dsorption } \\
\text { hedral angl } \\
\text { erpendicula }\end{array}$ & ance fro & Isorbate & & $\begin{array}{l}\text { bond length } \\
\text { of the oxy }\end{array}$ & $\mathrm{I}_{\mathrm{C}-\mathrm{C}} ; \mathrm{C}$ & bond le & $\mathrm{h}, \mathrm{d}_{\mathrm{C}-\mathrm{O}}$ \\
\hline
\end{tabular}


An electronic analysis was performed on the vertical guaiacol adsorption sites by examining the differential charge density distributions (Figure 4) and mean charge transfer between guaiacol and the surface upon guaiacol's vertical adsorption. The differential charge density distributions show that the charge transfer between vertical guaiacol and the $\mathrm{Fe}(110)$ and Pd (111) surfaces is negligible. The differential charge density distribution can be quantified using the mean charge transfer, which was found to be 0.12 and 0.28 electrons for vertical guaiacol on Fe (110) and $\mathrm{Pd}(111)$, respectively. These results are in agreement with the energetic and structural results shown in Table 2 and shows that vertically adsorbed guaiacol is merely physisorbed on both metal surfaces. Furthermore, these results are supported by the PDOS for these most favorable vertical sites, as both the metal's d-states and guaiacol's p-states remain unaffected by the vertical adsorption of guaiacol on either Pd (111) or Fe (110) (Figure S3).

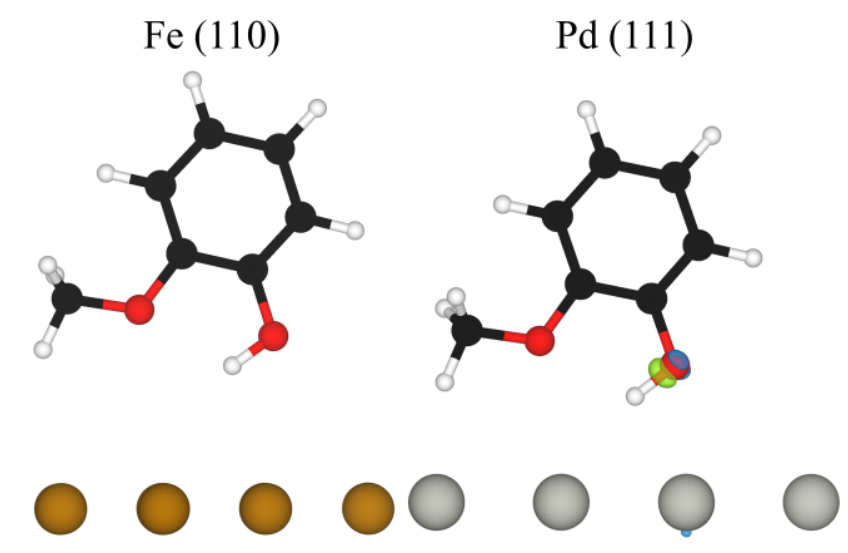

Figure 4. Differential charge density distributions for guaiacol vertically adsorbed on Fe (110) and Pd (111) in the most favorable adsorption sites. The sphere coloring is identical to Figure 3 and the charge density distribution isosurface was set to 0.005 electrons/Bohr ${ }^{3}$ with the green or blue colors representing a gain or loss of electrons, respectively. Calculations were performed with the optB88-vdW functional. 
Overall, these results show that vertical guaiacol physisorbs on both the Pd (111) and Fe (110) surfaces. This result was evident in the adsorption energy, molecular geometry, and electronic analyses. Furthermore, the physisorbed nature of vertical guaiacol on both surfaces is nearly identical to that of vertical phenol. Both phenol and guaiacol adsorbed vertically have nearly identical adsorption energies and mean charge transfer values on Fe (110) and Pd (111).[16] These results show that the addition of a second functional group, which is only weakly electron withdrawing, to the aromatic ring does not significantly alter the vertical adsorption of the phenolic compound. Additionally, the weak adsorption energy of the vertical site suggests that this configuration is not likely to be present under typical HDO conditions, and is unlikely to result in any significant catalysis. This is supported by our previous work,[17] where the cleavage of the $\mathrm{C}-\mathrm{O}$ bond in vertical phenol on the Fe surface does not occur until the horizontal adsorption phenol. As the horizontal adsorption of these phenolic compounds seems necessary in order for HDO to occur with reasonable activity, the HDO activity of a given metal surface will then be related to its oxophilicity, as shown by Resasco, et al.[42-44]

\subsection{Horizontal Guaiacol Adsorption}

The horizontal adsorption of guaiacol on the Fe (110) surface can occur with the aromatic ring being centered on the $3 \mathrm{~F}, \mathrm{LB}, \mathrm{SB}$, or $\mathrm{T}$ sites with the ring oriented either in line with the perpendicular Fe rows $\left(0^{\circ}\right)$ or rotated by $30^{\circ}$, identical to the adsorption sites for benzene on this surface.[15] Each of these ring positions has several rotations with respect to the placement of the hydroxyl and methoxy functional groups and each site is labeled based first on the hydroxyl group's placement (LB, $\mathrm{SB}, 3 \mathrm{~F}$, and $\mathrm{T})$ and second on the methoxy group's placement $(\mathrm{LB}, \mathrm{SB}$, 3F, and T). For guaiacol's horizontal adsorption on both Fe (110) and Pd (111), we used the most stable gas phase configuration (Figure 1a) as the adsorption of guaiacol in the second most 
favorable gas phase guaiacol configuration was found to be less energetically favorable (Figure S2). These adsorption sites are shown in Figure 5a and their corresponding adsorption energies (relative to gas phase guaiacol), calculated with the PW91 functional, are shown in Table 3.
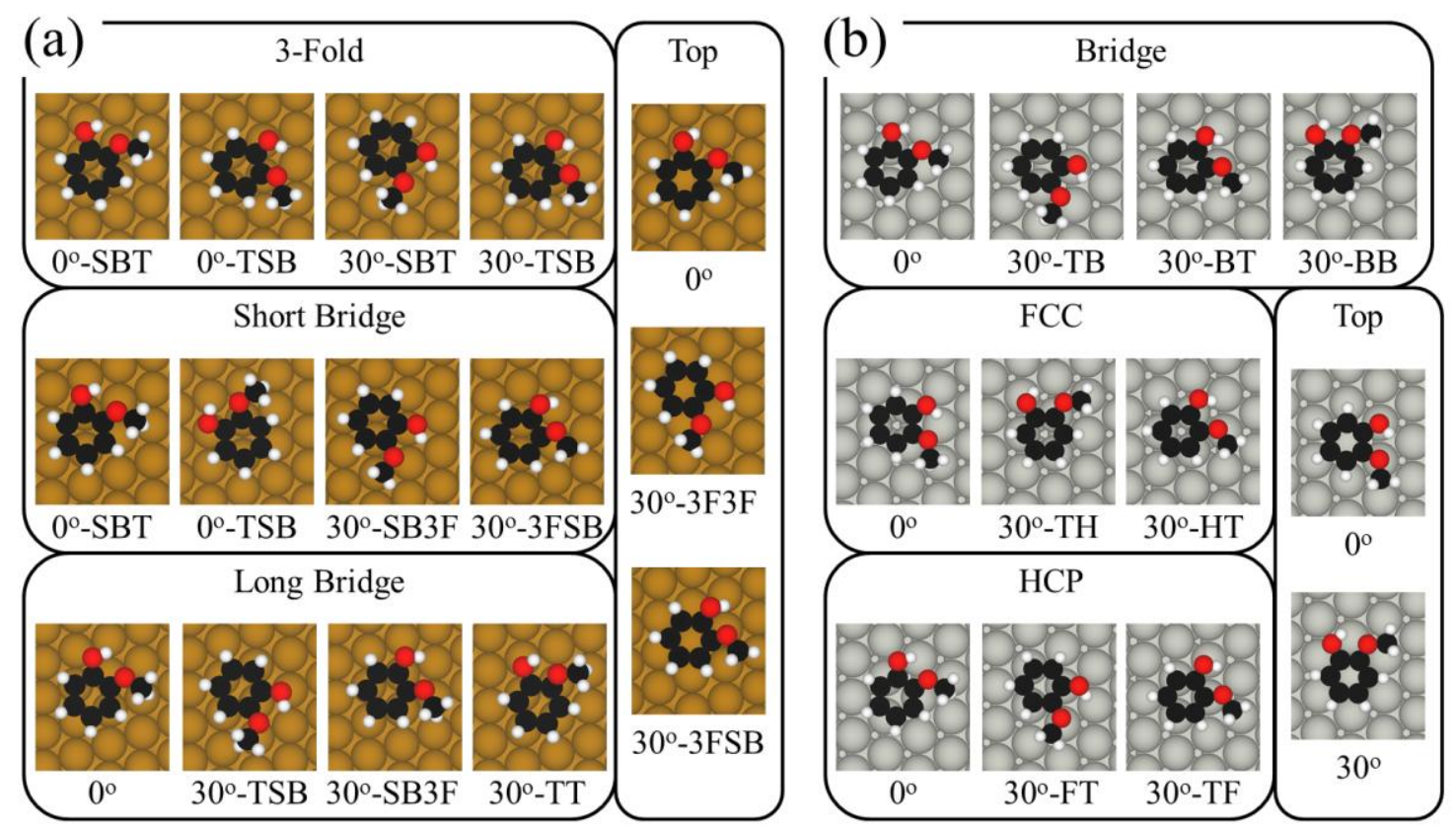

Figure 5. Horizontal adsorption sites for guaiacol on (a) Fe (110) and (b) Pd (111). The sphere coloring is identical to Figure 3.

Table 3. Adsorption energies for guaiacol (horizontal) on the Fe (110) and Pd (111) surfaces calculated with the PW91 functional. ${ }^{\mathrm{a}}$ The adsorption energies are shown in the order in which the structures appear in Figure 5. The $U$ symbol marks an adsorption site as unstable.

\begin{tabular}{|c|c|c|c|}
\hline \multicolumn{2}{|c|}{ Fe (110) } & \multicolumn{2}{|c|}{ Pd (111) } \\
\hline Site & $\mathrm{E}_{\mathrm{ads}}(\mathrm{eV})$ & Site & $\mathrm{E}_{\mathrm{ads}}(\mathrm{eV})$ \\
\hline 3-fold $0^{\circ}$ & $-0.82 /-0.84$ & bridge $0^{\circ}$ & $U$ \\
\hline 3-fold $30^{\circ}$ & $-0.88 /-0.85$ & bridge $30^{\circ}$ & $-1.05 /-1.03 /-1.14$ \\
\hline long bridge $0^{\circ}$ & -0.72 & $\operatorname{fcc} 0^{\circ}$ & -0.93 \\
\hline long bridge $30^{\circ}$ & $-0.87 /-0.89 /-0.96$ & fcc $30^{\circ}$ & $U / U$ \\
\hline short bridge $0^{\circ}$ & $-0.64 /-0.63$ & hep $0^{\circ}$ & -0.97 \\
\hline short bridge $30^{\circ}$ & $U / U$ & hcp $30^{\circ}$ & $U / U$ \\
\hline top $0^{\circ}$ & $U$ & top $0^{\circ}$ & -0.26 \\
\hline top $30^{\circ}$ & $U / U$ & top $30^{\circ}$ & -0.22 \\
\hline
\end{tabular}


The most favorable adsorption site for horizontal guaiacol on Fe (110) was found to be the LB30 $30^{\circ}$-TT site. This site has both oxygen groups atop surface Fe atoms and the bond lengths between the aromatic carbon and oxygen groups are $1.42 \AA$ and $1.41 \AA$ for the hydroxyl and methoxy groups, respectively. The $\mathrm{C}_{\text {aromatic }}-\mathrm{O}$ dihedral angles, relative to the plane of the aromatic ring, for this site were $17^{\circ}$ and $48^{\circ}$ for the hydroxyl and methoxy groups, respectively. Furthermore, the surface to adsorbate interactions cause the aromatic ring to distort with the average C-C bond length being $1.46 \AA$ and the average $\mathrm{C}$ - $\mathrm{H}$ dihedral angle being $23^{\circ}$.

The horizontal adsorption of guaiacol on the Pd (111) surface can occur with the aromatic ring being centered on the $\mathrm{B}, \mathrm{F}, \mathrm{H}$, or $\mathrm{T}$ sites with the ring oriented either in line with the perpendicular $\mathrm{Pd}$ rows $\left(0^{\circ}\right)$ or rotated by $30^{\circ}$, identical to the adsorption sites for benzene on this surface.[40] Each of these ring positions has several rotations with respect to the placement of the hydroxyl and methoxy functional groups and each site is labeled based first on the hydroxyl group's placement $(\mathrm{B}, \mathrm{F}, \mathrm{H}$, and $\mathrm{T})$ and second on the methoxy group's placement $(\mathrm{B}, \mathrm{F}, \mathrm{H}$, and T). These adsorption sites are shown in Figure $5 \mathrm{~b}$ and their corresponding adsorption energies (relative to gas phase guaiacol), calculated with the PW91 functional, are shown in Table 3.

The most favorable adsorption site for horizontal guaiacol on Pd (111) was found to be the $\mathrm{B} 30^{\circ}-\mathrm{BB}$ site. This site has both oxygen groups above surface bridge sites and the bond lengths between the aromatic carbon and oxygen groups are $1.37 \AA$ and $1.36 \AA$ for the hydroxyl and methoxy groups, respectively. The $\mathrm{C}_{\text {aromatic }}-\mathrm{O}$ dihedral angles, with respect to the horizontal plane of the aromatic ring, for this site were $21^{\circ}$ and $24^{\circ}$ for the hydroxyl and methoxy groups, respectively. Furthermore, the surface to adsorbate interactions cause the aromatic ring to distort with the average $\mathrm{C}-\mathrm{C}$ bond length being $1.45 \AA$ and the average $\mathrm{C}-\mathrm{H}$ dihedral angle being $22^{\circ}$. 
The most favorable horizontal guaiacol adsorption sites on Fe (110) and $\mathrm{Pd}(111)$ were studied further using the optB88-vdW functional and the resulting adsorption energies are shown in Table 2. The addition of the van der Waals corrections increased the adsorption energy of vertical guaiacol by $1.1 \mathrm{eV}$ for both surfaces, which is consistent with trends for other aromatic molecules.[15, 16, 31] The adsorption energy results show that guaiacol adsorbs stronger on Pd (111) than on Fe (110) by $0.3 \mathrm{eV}$. However, the distortion energy and geometric results show that guaiacol's structure is distorted to a greater degree by its interactions with the Fe (110) surface compared to the $\mathrm{Pd}$ (111) surface. Both the large adsorption and distortion energies for horizontal guaiacol suggest that this adsorption configuration results in chemisorbed guaiacol. Comparing the horizontal guaiacol adsorption results to that of horizontal phenol shows that the addition of the methoxy functional group does not significantly affect the adsorption energy of guaiacol on either surface.[16] The only significant change between horizontal phenol and guaiacol is the greater distortion energy for guaiacol on Fe (110).

An electronic analysis was performed on the horizontal guaiacol adsorption sites which examined the differential charge density distributions (Figure 6), mean charge transfer, and PDOS (Figure 7) upon guaiacol's horizontal adsorption. Additionally, the guaiacol orbitals most significantly involved in the horizontal configuration of guaiacol on both Fe (110) and Pd (111) were examined using the partial charge density distributions (Figure 8).

The differential charge density distributions (Figure 6) show that the charge transfer between horizontal guaiacol and the Fe (110) and Pd (111) surfaces is significant with the mean charge transfer for the Fe (110) and Pd (111) systems being 1.47 electrons and 1.68 electrons, respectively. This high degree of charge transfer between adsorbate and surface is consistent with chemisorption and is similar to that seen for phenol on both surfaces.[16] Upon guaiacol's 
adsorption on Fe (110) or Pd (111), guaiacol's highest energy orbitals will overlap with the metal's d-states, resulting in charge transfer and chemisorption. As shown in Figure 2, the highest energy orbitals of guaiacol contain the carbon p-states forming the $\pi$ cloud of charge around the ring for the aromatic carbon, as well as p-states for the oxygen functional groups. This suggests that the majority of the interaction between adsorbed guaiacol and the metal surfaces will be with these states, as seen clearly in Figure 6 where the majority of the charge transfer upon guaiacol adsorption occurs between the aromatic ring carbons and the metal surfaces. The Bader charge analysis supports this result (Table S2), showing that the aromatic carbons gain charge upon adsorption while the oxygen do not see a change in their charge. Furthermore, both the Fe and Pd surfaces lose charge upon guaiacol's adsorption with Fe being more significantly affected than Pd. The major difference between guaiacol horizontally adsorbed on $\mathrm{Fe}(110)$ and $\mathrm{Pd}(111)$ is the electronic interaction between the functional groups and the metal surfaces. On the $\mathrm{Fe}(110)$ surface, the hydroxyl oxygen has a significant interaction with the nearby Fe atom, which is similar to that seen for phenol on Fe (110).[16] On Pd (111), none of the species in the functional groups appear to interact with the surface atoms, which is different from the hydroxyl hydrogen to surface Pd interaction seen for phenol.[16] While the differential charge density for guaiacol on Pd (111) shows a small degree of charge transfer away from the methyl hydrogen nearest the Pd surface and the hydroxyl hydrogen, the Bader charge analysis (Table S2) shows no significant change to the charge on these atoms. 


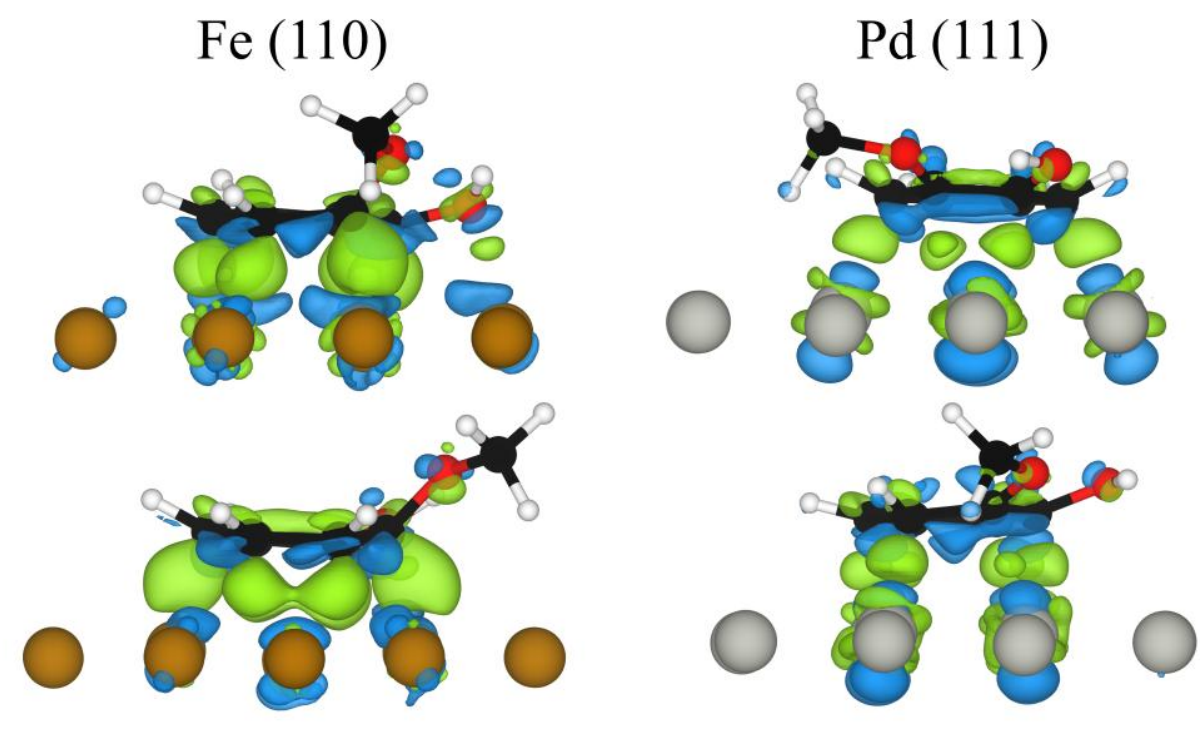

Figure 6. Differential charge density distributions for guaiacol horizontally adsorbed on Fe (110) and Pd (111) in the most favorable adsorption sites. Two different perspectives are given for each system to better visualize the charge transfer from the functional groups. The sphere coloring is identical to Figure 3 and the charge density distribution isosurface was set to 0.005 electrons/Bohr ${ }^{3}$ with the green or blue colors representing a gain or loss of electrons, respectively. Calculations were performed with the optB88-vdW functional.

Further details concerning the electronic effect of the adsorbate-surface interactions can be gleaned from an examination of the PDOS and partial charge density distributions, shown in Figure 7 and 8, respectively. The PDOS plotted in Figure 7 shows the d-states for the surface Fe and Pd as well as the p-states for the oxygen and carbon in guaiacol. For the metal states, the clean metal (Fe or Pd), metal bonded to aromatic carbon (Fe-C or $\mathrm{Pd}-\mathrm{C}$ ), and metal bonded to oxygen (Fe-O or $\mathrm{Pd}-\mathrm{O})$ are shown. For the carbon states, an aromatic carbon bonding to the metal surface $\left(\mathrm{C}_{\mathrm{a}}\right)$ and the methyl carbon $\left(\mathrm{C}_{\mathrm{m}}\right)$ are shown. For the oxygen states, both the hydroxyl $\left(\mathrm{O}_{\mathrm{h}}\right)$ and methoxy $\left(\mathrm{O}_{\mathrm{m}}\right)$ oxygen are shown. The atoms used for the PDOS plots in 
Figure 7 are shown in more detail in Figure S4. For the partial charge density distributions, the energy ranges used to calculate said distributions are shown in Table S1.
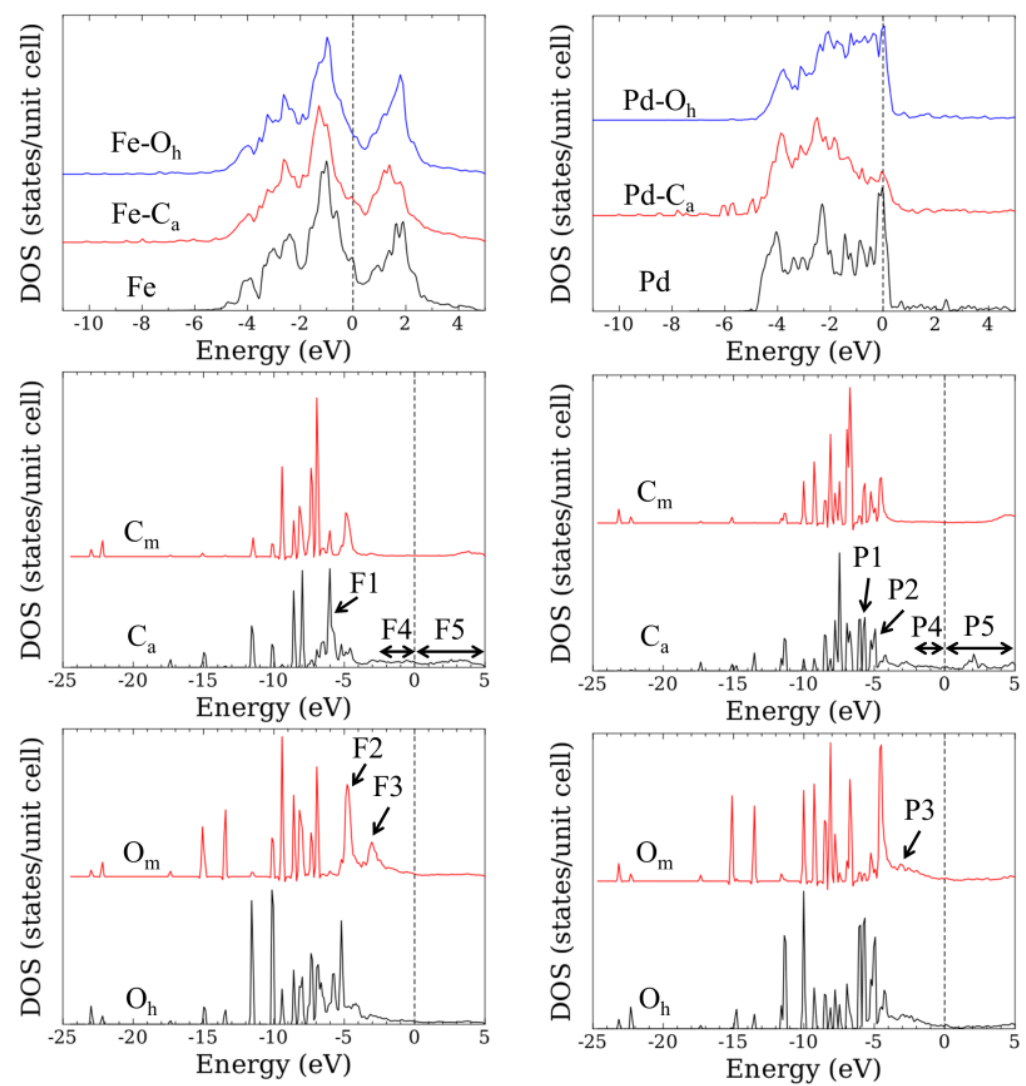

Figure 7. Partial density of states for guaiacol horizontally adsorbed on Fe (110) (left) and Pd (111) (right). The d-states are shown for the metals and the p-states are shown for both carbon and oxygen. The clean Fe surface, clean Pd surface, aromatic carbon, methyl carbon, hydroxyl oxygen, and methoxy oxygen are labeled as $\mathrm{Fe}, \mathrm{Pd}, \mathrm{C}_{\mathrm{a}}, \mathrm{C}_{\mathrm{m}}, \mathrm{O}_{\mathrm{h}}$, and $\mathrm{O}_{\mathrm{m}}$, respectively. The aromatic carbon bonded $\mathrm{Fe}(\mathrm{Fe}-\mathrm{C})$, aromatic carbon bonded $\mathrm{Pd}(\mathrm{Pd}-\mathrm{C})$, hydroxyl oxygen bonded Fe (Fe-O), and hydroxyl oxygen bonded Pd (Pd-O) are labeled in Figure S4 and the Fermi level has been set to zero.

The changes to the metal's (Fe or Pd) are minimal upon the adsorption of guaiacol on the surfaces. For the Pd (111) surface, the adsorption of guaiacol results in a shift in the d-states for 
the Pd atoms bonded to the aromatic carbon to lower energies while there is almost no change for the surface Pd nearest to the hydroxyl functional group. Comparing the d-band parameters (Table 4) for these different surface Pd atoms quantifies these changes to the Pd's d-states due to guaiacol adsorption and show that the pure $\mathrm{Pd}$ and $\mathrm{Pd}-\mathrm{O}$ states are nearly identical while the PdC state's d-band center has shifted by $0.4 \mathrm{eV}$ away from the Fermi level. This change to the Pd's d-states can also be seen in the partial charge density distributions (Figure 8) where there is significant overlap between the aromatic carbon and surface Pd's d-states and there is no apparent overlap between the surface Pd's charge density and either oxygen atom near the Fermi level. For the Fe (110) surface, both the Fe atoms bonded to aromatic carbon and the surface $\mathrm{Fe}$ atom nearest to, and bonding with, the guaiacol's hydroxyl oxygen are slightly shifted to lower energies. Comparing the d-band parameters for the clean, carbon, and oxygen bonded Fe atoms show that both the aromatic carbon and hydroxyl oxygen have a similar effect on the Fe's dstates, shifting their d-band center by $0.2 \mathrm{eV}$ away from the Fermi level after guaiacol's adsorption. An examination of the partial charge density distributions shows that there is significant overlap between the Fe's d-states and the aromatic carbon's p-states. Also, the partial charge density distribution at F3 (Figure 8) shows that there is a small degree of charge overlap between guaiacol's hydroxyl oxygen and the nearest surface Fe atom. 


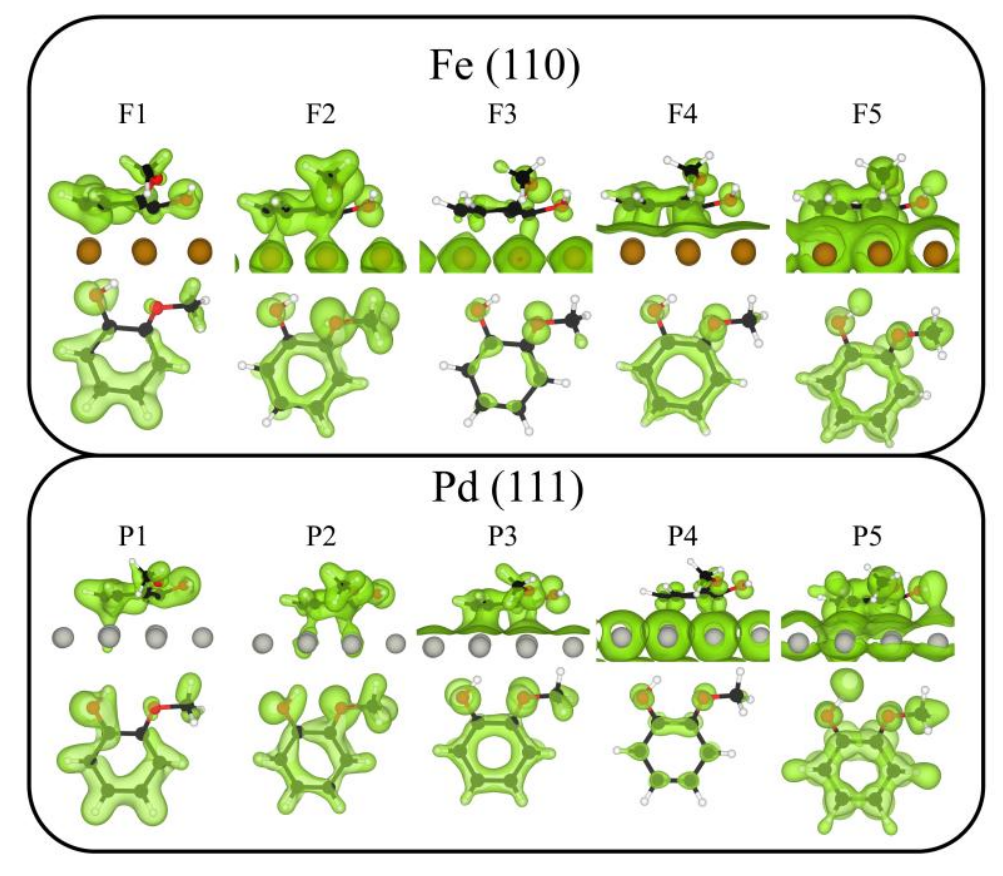

Figure 8. Top and side views of the partial charge density distributions of the highest energy orbitals for guaiacol on Fe (110) in the $\mathrm{LB}^{\circ} 0^{\circ}$-TT site and on $\mathrm{Pd}(111)$ in the $\mathrm{B} 30^{\circ}$-BB site. The labels for each distribution correspond to Figure 7 . The isosurface was set at $0.01 \mathrm{e} / \mathrm{Bohr}^{3}$ and the green areas represent the presence of charge. The calculations were performed with the optB88vdW functional and the sphere coloring is identical to Figure 3.

Table 4. d-band center and d-band width on the metal surface atoms involved in the horizontal adsorption of guaiacol on Fe (110) and Pd (111). The labels used here are the same as Figure 7 and these calculations used the optB88-vdW functional.

\begin{tabular}{ccc}
\hline \multicolumn{3}{c}{ Fe (110) } \\
\hline Site & d-band center (eV) & d-band width (eV) \\
Fe & -1.8 & 2.1 \\
$\mathrm{Fe}-\mathrm{C}$ & -2.0 & 2.5 \\
$\mathrm{Fe}-\mathrm{O}$ & -2.0 & 2.4 \\
\hline \multicolumn{3}{c}{ Pd (111) } \\
\hline Site & d-band center (eV) & d-band width (eV) \\
Pd & -1.8 & 2.2 \\
Pd-C & -2.2 & 2.6 \\
Pd-O & -1.9 & 2.2
\end{tabular}


The only major changes to the carbon p-states upon guaiacol's adsorption on both Fe (110) and Pd (111) occur in the aromatic carbon while the methyl carbon appears largely unaffected by the adsorption. For the aromatic carbon species, on both the Fe (110) and Pd (111) surfaces the gas phase guaiacol's LUMO and HOMO peaks largely disappear as these states shift to lower energies and the charge in these orbitals become delocalized due to the interaction between the aromatic carbon and the surface metal atoms. This is evident from the partial charge density distributions (Figure 8) which show that the major charge interaction between the surfaces and the aromatic carbon occur in energies between $\sim-4 \mathrm{eV}$ and the Fermi level, which has no significant peak in the PDOS for either surface. For the methyl carbon species, no significant changes in the PDOS are evident upon guaiacol's adsorption compared to gas phase guaiacol. Both the PDOS and partial charge density distributions show no interaction between the methyl carbon's p-states and both the Fe (110) and Pd (111) surfaces.

The largest difference in the electronic structure of guaiacol adsorbed on Fe (110) and Pd (111) can be seen in the oxygen p-states. For the hydroxyl oxygen, the HOMO and LUMO states are delocalized, similar to the aromatic carbon p-states, on both Fe (110) and Pd (111). The major difference between the Fe (110) and Pd (111) surfaces for the hydroxyl oxygen is the small degree of charge overlap between the oxygen atom and a surface atom on the $\mathrm{Fe}$ (110) surface at F3 (Figure 8) with negligible overlap seen between the hydroxyl oxygen and the Pd (111) surface (Figure 8 orbital P3). For the methoxy oxygen, there are only minor changes when guaiacol is adsorbed on $\mathrm{Fe}$ (110) compared to gas phase guaiacol, with two distinct peaks observed at F3 and F2 (Figure 7) that correspond to G3 and G2 (Figure 2), while the change to the methoxy oxygen on Pd (111) is similar to that seen for the hydroxyl oxygen (Figure 7). This difference in the methoxy oxygen's states on Fe (110) and Pd (111) can be better understood in 
terms of the distortion of the methoxy group away from the surface, as the methoxy group has a significantly higher $\mathrm{C}_{\mathrm{a}}-\mathrm{O}_{\mathrm{m}}$ dihedral angle on $\mathrm{Fe}$ (110) as compared to $\mathrm{Pd}(111)$, as shown in Table 2. The greater distortion of the methoxy group on Fe (110) significantly reduces the interaction between the methoxy functional group and the surface atoms compared to the Pd (111) surface. Overall, the oxygen PDOS results show a similar change for the hydroxyl oxygen on Fe (110), the hydroxyl oxygen on Pd (111), and the methoxy oxygen on Pd (111) with the delocalization of the oxygen p-states at energies above $\sim-4 \mathrm{eV}$, while the increased distortion of the methoxy oxygen away from the Fe (110) surface results in the retention of this oxygen's pstates. However, the differential and partial charge density distributions show that the surface's distortion of the oxygen's p-states is most significant for the hydroxyl oxygen on Fe (110), with an observable overlap between the hydroxyl oxygen's p-states and the Fe's d-states.

Comparing guaiacol's PDOS and partial charge density distributions to that of phenol on Fe (110) and Pd (111),[16] it is clear that similar changes occur for both phenols. The metal states for Fe and Pd and the aromatic carbon states have similar shifts upon phenol and guaiacol adsorption. The similarity between guaiacol's and phenol's surface to adsorbate interactions upon adsorption on $\mathrm{Fe}(110)$ and $\mathrm{Pd}$ (111) show that the addition of weakly electron withdrawing functional groups on the aromatic ring do not significantly alter the adsorption of phenols, similar to that shown by Jenkins[21] and Bonalumi, et al.[45]

Overall, these results show that guaiacol adsorbs slightly stronger on the Pd (111) surface as compared to the $\mathrm{Fe}(110)$ surface. However, the $\mathrm{C}_{\text {aromatic }}-\mathrm{O}$ bonds are distorted to a greater degree on the Fe (110) surface. This result is similar to the trend seen for phenol adsorbing on Fe (110) and $\mathrm{Pd}(111) .[16]$ The electronic analyses show that there is an overlap between the hydroxyl oxygen's states in guaiacol and the Fe (110) surface while there is negligible 
interaction between the functional groups and the surface atoms when examining the adsorption of guaiacol on $\mathrm{Pd}$ (111). This result is qualitatively consistent with experimental results for the HDO of guaiacol on Fe and Pd catalysts which shows that Fe promotes the removal of oxygen functional groups while Pd promotes the saturation of the aromatic ring.[10,11]

\section{Conclusions}

The adsorption of guaiacol on Fe (110) and Pd (111) was studied using density functional theory. Both horizontal and vertical sites were tested and the adsorption energy results showed that the vertical sites were merely physisorbed while the horizontal sites were chemisorbed. The Pd (111) surface was seen to adsorb horizontal guaiacol slightly stronger than Fe (110). However, the Fe (110) surface produced a greater distortion of the $\mathrm{C}-\mathrm{O}$ bonds in the adsorbed guaiacol than the Pd (111) surface. These energetic and geometric results were caused by the greater degree of charge transfer between guaiacol and $\mathrm{Pd}(111)$ while further electronic analyses showed that the hydroxyl oxygen's p-states overlap to a small degree with the surface Fe (110) states. Furthermore, no obvious interaction between the Pd (111) states and guaiacol's functional groups was observed. Overall, this work has shown that the Fe (110) surface has a greater effect on guaiacol's functional groups due to the more pronounced electronic interactions between the oxygen and surface Fe while the Pd (111) has a stronger interaction between the surface Pd and the aromatic ring.

\section{Supporting Information}

The Supporting Information contains: a comparison of the adsorption of two different gas phase guaiacol configurations on Fe (110) in the vertical and horizontal situations; the PDOS od every carbon and oxygen atom in gas phase guaiacol; the PDOS for the most favorable vertical guaiacol sites on Fe (110) and Pd (111); a figure showing the metal atoms used for the metal 
PDOS in the horizontal guaiacol sites on Fe (110) and Pd (111); the energy ranges used to obtain the partial charge density distributions; and the Bader charges on guaiacol adsorbed horizontally on Fe (110) and Pd (111).

\section{Acknowledgements}

This work was supported by institutional funds provided to J.S.M. from the Voiland School of Chemical Engineering and Bioengineering and was partially funded by USDA/NIFA through Hatch Project \#WNP00807 titled: "Fundamental and Applied Chemical and Biological Catalysts to Minimize Climate Change, Create a Sustainable Energy Future, and Provide a Safer Food Supply". Our thanks also go to the donors of The American Chemical Society Petroleum Research Fund for partial support of this research. We acknowledge computational resources provided by the Center for Nanoscale Materials at Argonne National Laboratory. Use of the Center for Nanoscale Materials was supported by the U. S. Department of Energy, Office of Science, and Office of Basic Energy Sciences under Contract No. DE-AC02-06CH11357. This material is based upon work supported by the U.S. Department of Energy, Office of Science, Office of Workforce Development for Teachers and Scientists, Office of Science Graduate Student Research (SCGSR) program. The SCGSR program is administered by the Oak Ridge Institute for Science and Education for the DOE under contract number DE-AC05-06OR23100.

This work was also partially supported by U. S. Department of Energy (DOE), Office of Basic

Energy Sciences, Division of Chemical Sciences, Biosciences and Geosciences under Award Numbers DE-SC0014560 and DE-FG02-05ER15712.

\section{References}

[1] W. M. H. Sachtler, J. Mol. Catal. 25 (1984) 1-12.

[2] S. H. Oh, J. E. Carpenter, J. Catal. 98 (1986) 178-190.

[3] K. B. Arcuri, L. H. Schwartz, R. D. Piotrowski, J. B. Butt, J. Catal. 85 (1984) 349-361.

[4] M. P. Hogarth, G. A. Hards, Platinum Met. Rev. 40 (1996) 150-159. 
[5] G. A. Somorjai, C. Aliaga, Langmuir 26 (2010) 16190-203.

[6] K. An, G. A. Somorjai, Catalysis Letters 145 (2014) 233-248.

[7] S. Alayoglu, G. A. Somorjai, Catalysis Letters 145 (2014) 249-271.

[8] G. A. Somorjai, H. Frei, J. Y. Park, J. Am. Chem. Soc. 131 (2009) 16589-16605.

[9] F. Tao, M. E. Grass, Y. Zhang, D. R. Butcher, F. Aksoy, S. Aloni, V. Altoe, S. Alayoglu, J.

R. Renzas, C.-K. Tsung, Z. Zhu, Z. Liu, M. Salmeron, G. A. Somorjai, J. Am. Chem. Soc. 132 (2010) 8697-8703.

[10] J. Sun, A. M. Karim, H. Zhang, L. Kovarik, X. Li, A. J. Hensley, J.-S. McEwen, Y. Wang, J. Catal. 306 (2013) 47-57.

[11] Y. Hong, H. Zhang, J. Sun, A. M. Karim, A. J. Hensley, M. Gu, M. H. Engelhard, J.-S. McEwen, Y. Wang, ACS Catal. 4 (2014) 3335-3345.

[12] L. Nie, P. M. de Souza, F. B. Noronha, W. An, T. Sooknoi, D. E. Resasco, J. Mol. Catal. A 388-389 (2014) 47-55.

[13] S. Sitthisa, W. An, D. E. Resasco, J. Catal. 284 (2011) 90-101.

[14] R. N. Olcese, M. Bettahar, D. Petitjean, B. Malaman, F. Giovanella, A. Dufour, Appl. Catal. B 115 (2012) 63-73.

[15] A. J. Hensley, R. Zhang, Y. Wang, J.-S. McEwen, J. Phys. Chem. C 117 (2013) 2431724328.

[16] A. J. R. Hensley, Y. Wang, J.-S. McEwen, Surf. Sci. 630 (2014) 244-253.

[17] A. J. Hensley, Y. Wang, J.-S. McEwen, ACS Catal. 5 (2015) 523-536.

[18] A. J. Hensley, Y. Hong, R. Zhang, H. Zhang, J. Sun, Y. Wang, J.-S. McEwen, ACS Catal. 4 (2014) 3381-3392.

[19] D. R. Strongin, G. A. Somorjai, J. Catal. 109 (1988) 51-60.

[20] D. R. Strongin, S. R. Bare, G. A. Somorjai, J. Catal. 103 (1987) 289-301.

[21] S. J. Jenkins, Proc. Roy. Soc. A 465 (2009) 2949-2976.

[22] G. Kresse, J. Furthmueller, Phys. Rev. B 54 (1996) 11169-11186.

[23] G. Kresse, J. Hafner, Phys. Rev. B 47 (1993) 558-561.

[24] G. Kresse, D. Joubert, Phys. Rev. B 59 (1999) 1758-1775.

[25] P. E. Blöchl, Phys. Rev. B 50 (1994) 17953-17979.

[26] J. P. Perdew, K. A. Jackson, M. R. Pederson, D. J. Singh, C. Fiolhais, Phys. Rev. B 46 (1992) 6671-6687.

[27] J. P. Perdew, Y. Wang, Phys. Rev. B 45 (1992) 13244-13249.

[28] J. Klimeš, D. R. Bowler, A. Michaelides, Phys. Rev. B 83 (2011) 195131.

[29] Y. Yoon, R. Rousseau, R. S. Weber, D. Mei, J. A. Lercher, J. Am. Chem. Soc. 136 (2014) 10287-10298.

[30] W. Liu, V. G. Ruiz, G.-X. Zhang, B. Santra, X. Ren, M. Scheffler, A. Tkatchenko, New J. Phys. 15 (2013) 053046.

[31] W. Liu, J. Carrasco, B. Santra, A. Michaelides, M. Scheffler, A. Tkatchenko, Phys. Rev. B 86 (2012) 245405.

[32] B. Hammer, L. B. Hansen, J. K. Nørskov, Phys. Rev. B 59 (1999) 7413-7421.

[33] J. P. Perdew, K. Burke, M. Ernzerhof, Phys. Rev. Lett. 77 (1996) 3865-3868.

[34] F. Mittendorfer, J. Hafner, Surf. Sci. 472 (2001) 133-153.

[35] G. Santarossa, M. Iannuzzi, A. Vargas, A. Baiker, ChemPhysChem 9 (2008) 401-413.

[36] L. Ghiringhelli, R. Caputo, L. Delle Site, Phys. Rev. B 75 (2007) 113403.

[37] R. F. W. Bader, Atoms in Molecules: A Quantum Theory. 1990, Oxford: Oxford University Press. 
[38] J. R. Kitchin, J. K. Nørskov, M. A. Barteau, J. G. Chen, J. Chem. Phys. 120 (2004) 1024010246.

[39] X. Sun, Y. Yamauchi, M. Kurahashi, T. Suzuki, Z. P. Wang, S. Entani, J. Phys. Chem. C 111 (2007) 15289-15298.

[40] H. Orita, N. Itoh, Appl. Catal. A 258 (2004) 17-23.

[41] C. Morin, D. Simon, P. Sautet, J. Phys. Chem. B 108 (2004) 5653-5665.

[42] P. M. de Souza, L. Nie, L. E. P. Borges, F. B. Noronha, D. E. Resasco, Catal. Lett. 144 (2014) 2005-2011.

[43] P. M. de Souza, R. C. Rabelo-Neto, L. E. P. Borges, G. Jacobs, B. H. Davis, T. Sooknoi, D. E. Resasco, F. B. Noronha, ACS Catal. 5 (2015) 1318-1329.

[44] Q. Tan, G. Wang, L. Nie, A. Dinse, C. Buda, J. Shabaker, D. E. Resasco, ACS Catal. (2015).

[45] N. Bonalumi, A. Vargas, D. Ferri, A. Baiker, J. Phys. Chem. B 110 (2006) 9956-9965. 

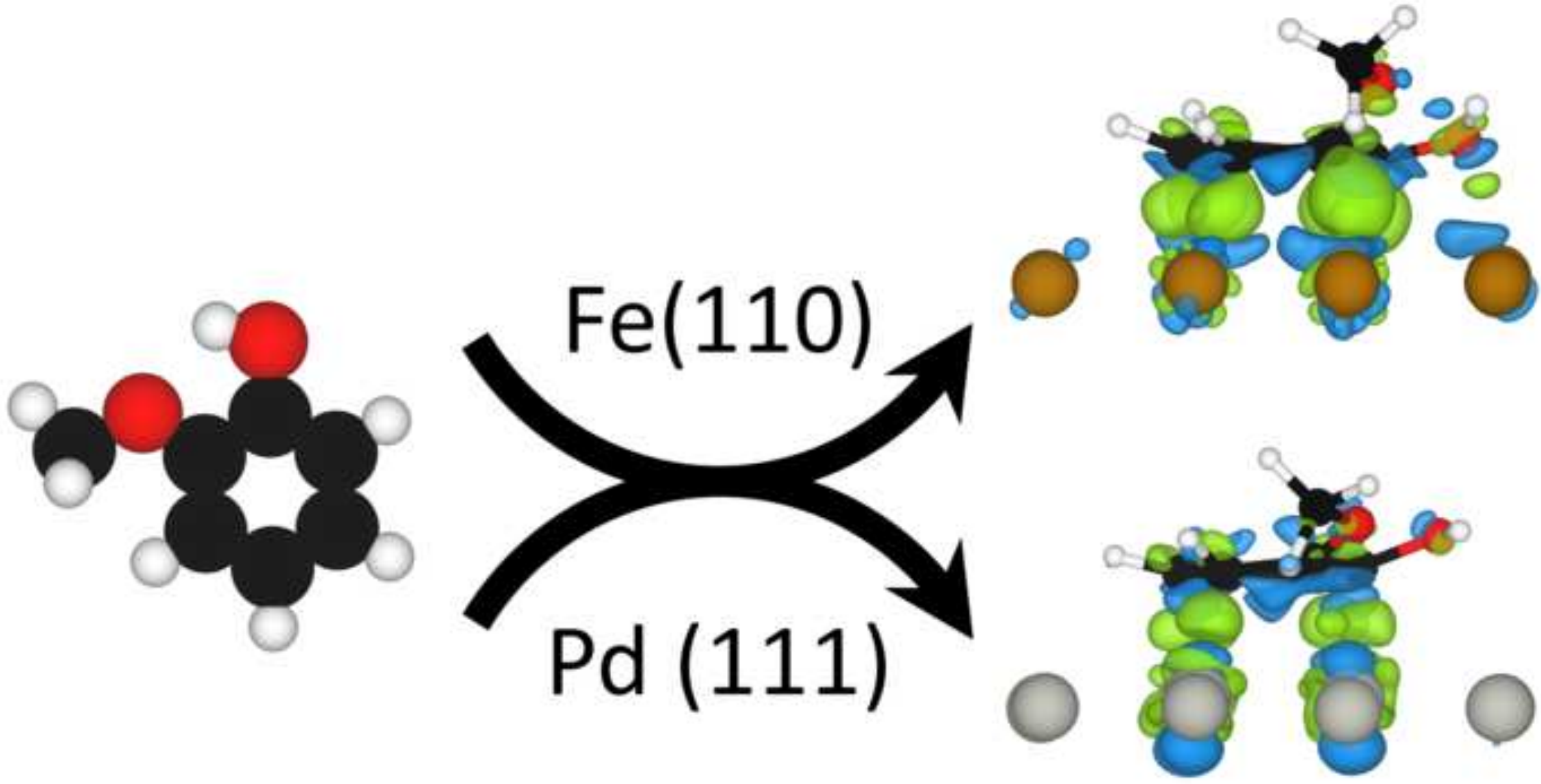